\title{
FREE VIBRATION OF COMPOSITE CIRCULAR MEMBRANES
}

\author{
Urszula Siedlecka, Stanistaw Kukla, Izabela Zamorska
}

Institute of Mathematics, Czestochowa University of Technology, Poland urszula.siedlecka@im.pcz.pl,stanislaw.kukla@im.pcz.pl, izabela.zamorska@im.pcz.pl

\begin{abstract}
In this paper, the object of consideration is a membrane consisting of one circular and two annular segments. The Green's functions method for the free vibration of this membrane is applied. A numerical example illustrating this method is given.
\end{abstract}

\section{Introduction}

The determination of vibrational characteristics of mechanical structures is an important problem in various engineering fields. In particular, the eigenfrequencies of membranes have great significance to the design of aeronautical, naval and civil structures as well as musical instruments. The free vibration of composite, circular annular membranes are the subject of papers [1-3].

The solution to the vibration problem of a composite membrane consisting of two concentric membrane segments (each with constant density), can be derived in an exact form $[1,2]$. In this case the membrane segments create the nonhomogenous membrane for which the frequency equation by determinant of a $4 \times 4$ matrix is expressed. Using this equation in paper [1] the eigenfrequencies of the membrane for various ratio of membrane densities and various ratio of radii of the distinguished membrane segments were numerically determined. The general formulation of the eigenproblem for membrane of arbitrary discontinuous variations of the density in the radial direction is given in paper [2]. The presented numerical example also deals with the double connected membrane. In paper [3] the natural frequencies of the composite, doubly connected membrane obtained by using the finite element method are compared with the exact values. Examples of the application of the Green's function method to vibration problems of homogenous membranes are presented in book [4].

The separation time and space variables in the differential equation of the transverse vibration of a membrane leads to the Helmholtz equation. In the case of composite membrane consisting of many membrane segments of constant densities, the Helmholtz equations oblige in each segment. The continuity conditions are satisfied at the concentric circles which separate the segments of constant densities. The solution of this eigenproblem can be derived by using the Green's func- 
tion properties. The Green's functions which occur in the solution were derived in paper [5].

The present paper deals with the free vibration problem to a composite membrane consisting of three membrane segments of constant densities. The solution is obtained by using the Green's function method. The symbolic and numerical calculations were performed by the use of Mathematica [6].

\section{Formulation of the problem}

Consider the free vibration of a membrane consisting of one circular and two annular segments as shown in Figure 1. The differential equation for the displacement $u_{i}$ of the $i$-th membrane segment is

$$
s \nabla^{2} u_{i}-\rho_{i} \frac{\partial^{2} u_{i}}{\partial t^{2}}=0, \quad i=0,1,2
$$

where $s$ is the tension per unit lenght, $\rho_{i}$ is the density of $i$-th segment of the membrane, $\nabla^{2}=\frac{\partial^{2}}{\partial r^{2}}+\frac{1}{r} \frac{\partial}{\partial r}+\frac{1}{r^{2}} \frac{\partial^{2}}{\partial \theta^{2}}$ where $r, \theta$ are polar coordinates and $t$ is time.

The step-wise changes of densities of the membrane are at circles with radii $r_{0}$ and $r_{1}$. These circles determine the uniform segments of the considered membrane. At the distinguished circles the following continuity conditions are satisfied:

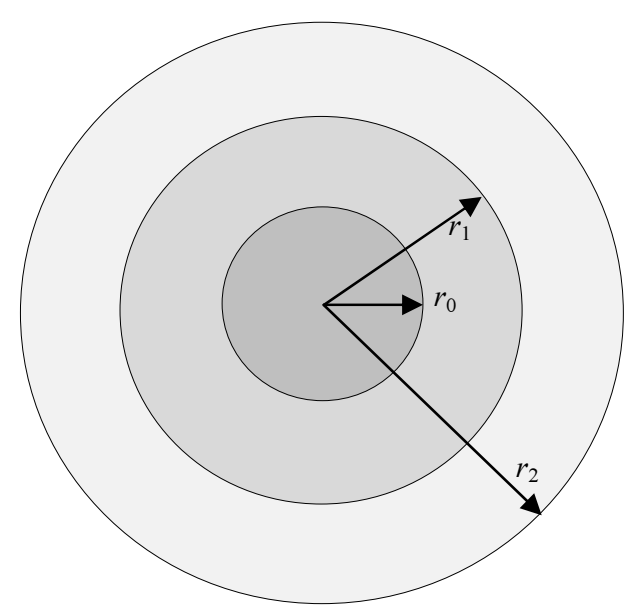

Fig. 1. A sketch of membrane under study 


$$
\begin{gathered}
u_{0}\left(r_{0}, \theta, t\right)=u_{1}\left(r_{0}, \theta, t\right), \quad u_{1}\left(r_{1}, \theta, t\right)=u_{2}\left(r_{1}, \theta, t\right) \\
\left.\frac{\partial u_{0}(r, \theta, t)}{\partial r}\right|_{r=r_{0}}=\left.\frac{\partial u_{1}(r, \theta, t)}{\partial r}\right|_{r=r_{0}},\left.\quad \frac{\partial u_{1}(r, \theta, t)}{\partial r}\right|_{r=r_{1}}=\left.\frac{\partial u_{2}(r, \theta, t)}{\partial r}\right|_{r=r_{1}}
\end{gathered}
$$

Moreover, the functions $u_{0}$ and $u_{2}$ satisfied the conditions

$$
\left|u_{0}(0, \theta, t)\right|<\infty, \quad u_{2}\left(r_{2}, \theta, t\right)=0
$$

The mode shapes of vibration of the membrane are obtained by using the classical method of separation of variables to equations (1)-(3). The modes of vibrations can be written as

$$
u_{i}(r, \theta, t)=U_{\text {in }}(r) \cos n \theta \cos \omega t
$$

where $\omega$ is the natural frequency of the membrane and $n$ is an integer. The function $U_{\text {in }}$ occurring in the above equation is a solution of the eigenproblem derived by substitution (4) into equations (1)-(3):

$$
\begin{gathered}
\frac{1}{r} \frac{d}{d r}\left(r \frac{d U_{i n}(r)}{d r}\right)+\left(\lambda_{i}^{2}-\frac{n^{2}}{r^{2}}\right) U_{i n}(r)=0 \quad i=0,1,2 \\
U_{0 n}\left(r_{0}\right)=U_{1 n}\left(r_{0}\right), \quad U_{1 n}\left(r_{1}\right)=U_{2 n}\left(r_{1}\right) \\
\left.\frac{d U_{0 n}(r)}{d r}\right|_{r=r_{0}}=\left.\frac{d U_{1 n}(r)}{d r}\right|_{r=r_{0}},\left.\quad \frac{d U_{1 n}(r)}{d r}\right|_{r=r_{1}}=\left.\frac{d U_{2 n}(r)}{d r}\right|_{r=r_{1}} \\
U_{0 n}(0)<\infty, U_{2 n}\left(r_{2}\right)=0
\end{gathered}
$$

where $\lambda_{i}=\omega \sqrt{\frac{\rho_{i}}{s}}$.

\section{Solution to the problem}

The solution to the problem is obtained by using the properties of the Green's function [4]. The Green's functions $G_{i}(i=0,1,2)$ which are necessary in this approach, satisfy differential equations $(\delta(\cdot)$ is the Dirac delta function)

$$
\frac{1}{r} \frac{\partial}{\partial r}\left(r \frac{\partial G_{i}(r, \zeta)}{\partial r}\right)+\left(\lambda_{i}^{2}-\frac{n^{2}}{r^{2}}\right) G_{i}(r, \zeta)=\frac{\delta(r-\zeta)}{r}, i=0,1,2
$$


and the homogeneous boundary conditions:

$$
\begin{aligned}
& \left.\frac{\partial}{\partial r} G_{0}(r, \zeta)\right|_{r=r_{0}}=0 \\
& \left.\frac{\partial}{\partial r} G_{1}(r, \zeta)\right|_{r=r_{0}}=\left.\frac{\partial}{\partial r} G_{1}(r, \zeta)\right|_{r=r_{1}}=0 \\
& \left.\frac{\partial}{\partial r} G_{2}(r, \zeta)\right|_{r=r_{1}}=0,\left.\quad G_{2}(r, \zeta)\right|_{r=r_{2}}=0
\end{aligned}
$$

On the basis of equation (5), which corresponds to the uniform membrane segment, the following equation can be written

$$
\int_{r_{i-1}}^{r_{i}}\left[\frac{d}{d \zeta}\left(\zeta \frac{d U_{i n}(\zeta)}{d \zeta}\right)+\zeta\left(\lambda_{i}^{2}-\frac{n^{2}}{\zeta^{2}}\right) U_{\text {in }}(\zeta)\right] G_{i}(r, \zeta) d \zeta=0, i=0,1,2
$$

where $r_{-1}=0$. Integrating by parts, one obtains

$$
\begin{gathered}
\int_{r_{i-1}}^{r_{i}}\left[\frac{\partial}{\partial \zeta}\left(\zeta \frac{\partial G_{i}(r, \zeta)}{\partial \zeta}\right)+\zeta\left(\lambda_{i}^{2}-\frac{n^{2}}{\zeta^{2}}\right) G_{i}(r, \zeta)\right] U_{i n}(\zeta) d \zeta \\
\quad=\left.\left(-\zeta \frac{d U_{i n}(\zeta)}{d \zeta} G_{i}(r, \zeta)+\zeta U_{i n}(\zeta) \frac{\partial G_{i}(r, \zeta)}{\partial \zeta}\right)\right|_{\zeta=r_{i-1}} ^{\zeta=r_{i}}
\end{gathered}
$$

Taking into account the equation (9) in the left-hand side of equation (12), we have

$$
\int_{r_{i-1}}^{r_{i}} \delta(r-\zeta) U_{i n}(\zeta) d \zeta=\left.\zeta\left(-G_{i}(r, \zeta) \frac{d U_{i n}(\zeta)}{d \zeta}+U_{i n}(\zeta) \frac{\partial G_{i}(r, \zeta)}{\partial \zeta}\right)\right|_{\zeta=r_{i-1}} ^{\zeta=r_{i}}
$$

Using the property of the Dirac delta function

$$
\int_{a}^{b} \delta(r-\zeta) U_{i n}(\zeta) d \zeta=U_{i n}(r) \quad \text { for } a \leq r \leq b
$$

in equation (13) and the boundary conditions (6)-(8) and (10), we obtain for $i=0,1,2$, successively

$$
\begin{array}{ll}
U_{0 n}(r)=S_{0 n} G_{0 n}\left(r, r_{0}\right), & 0 \leq r \leq r_{0} \\
U_{1 n}(r)=-S_{0 n} G_{1 n}\left(r, r_{0}\right)+S_{1 n} G_{1 n}\left(r, r_{1}\right), & r_{0}<r \leq r_{1} \\
U_{2 n}(r)=-S_{1 n} G_{2 n}\left(r, r_{1}\right), & r_{1}<r \leq r_{2}
\end{array}
$$


where $S_{0 n}=\left.r_{0} \frac{d U_{0 n}(r)}{d r}\right|_{r=r_{0}}, S_{1 n}=\left.r_{1} \frac{d U_{1 n}(r)}{d r}\right|_{r=r_{1}}$. Next the use of boundary conditions (6) leads to a system of equations:

$$
\left\{\begin{array}{l}
S_{0 n}\left[G_{0 n}\left(r_{0}, r_{0}\right)+G_{1 n}\left(r_{0}, r_{0}\right)\right]-S_{1 n} G_{1 n}\left(r_{0}, r_{1}\right)=0 \\
-S_{0 n} G_{1 n}\left(r_{1}, r_{0}\right)+S_{1 n}\left[G_{1 n}\left(r_{1}, r_{1}\right)+G_{2 n}\left(r_{1}, r_{1}\right)\right]=0
\end{array}\right.
$$

A nontrivial solution of this equation system (with respect to $S_{0 n}, S_{1 n}$ ) exists for these values of parameter $\omega$, which are roots of equation

$$
W_{n}\left(\Omega_{n}\right)=\left|\begin{array}{cc}
G_{0 n}\left(r_{0}, r_{0}\right)+G_{1 n}\left(r_{0}, r_{0}\right) & -G_{1 n}\left(r_{0}, r_{1}\right) \\
-G_{1 n}\left(r_{1}, r_{0}\right) & G_{1 n}\left(r_{1}, r_{1}\right)+G_{2 n}\left(r_{1}, r_{1}\right)
\end{array}\right|=0
$$

This equation is solved numerically with respect to the frequency parameter $\Omega_{n}=\omega_{n} r_{2} \sqrt{\frac{\rho_{2}}{s}}$. A graph of the function $W_{n}(\Omega)$ for $n=0, r_{0}=0.2, r_{1}=0.4, r_{2}=1.0$, $\sigma_{1}=1.0, \sigma_{2}=3.0$ is presented on Figure 2. We can observe the four roots of equation (17) on this figure.

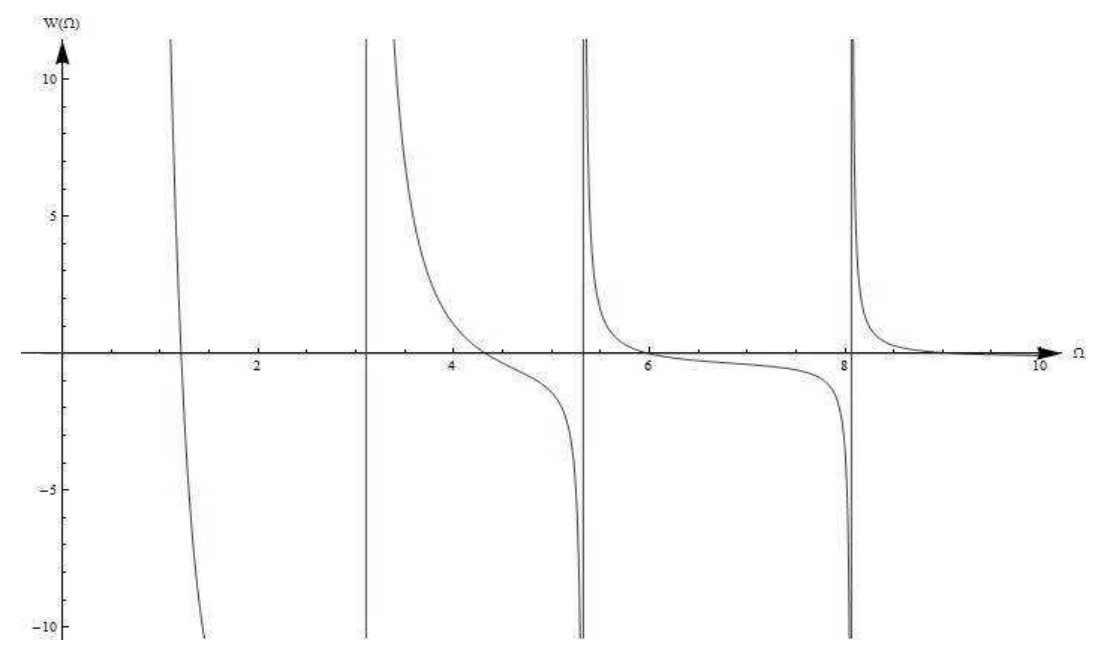

Fig. 2. A graph of function $W_{n}(\Omega)$ for $n=0$

The mode shapes of vibration corresponding to the determined frequencies are given by equations (15) where $S_{1 n}$ can be obtained on the basis of equation (16a)

$$
S_{1 n}=S_{0 n} \frac{G_{0 n}\left(r_{0}, r_{0}\right)+G_{1 n}\left(r_{0}, r_{0}\right)}{G_{1 n}\left(r_{0}, r_{1}\right)}
$$



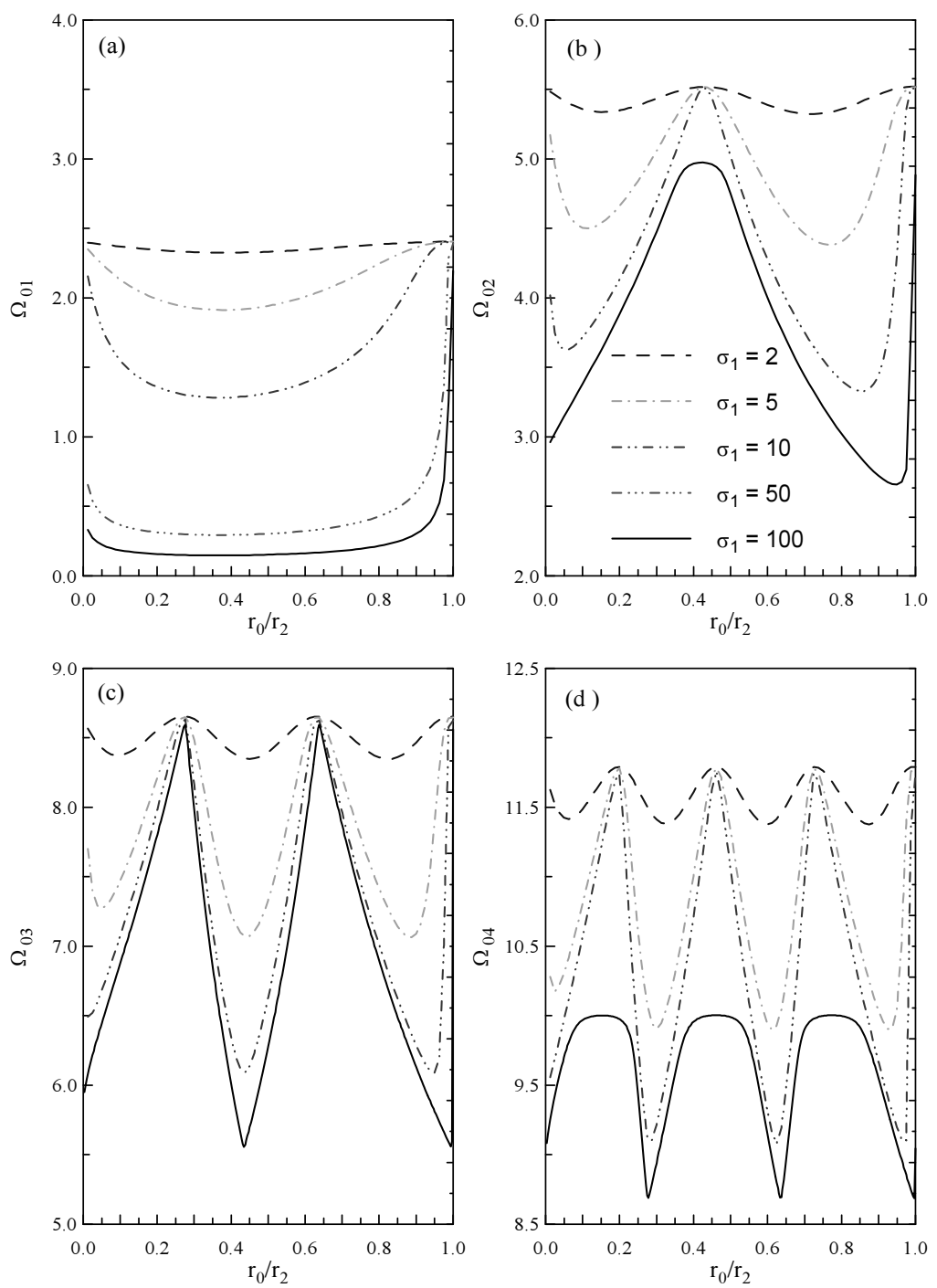

Fig. 3. Frequency parameter values $\Omega_{0 i},(i=1,2,3,4)$ as functions of the ratio $\frac{r_{0}}{r_{2}}$ for various values of $\sigma_{1}=\frac{\rho_{1}}{\rho_{2}}$

\section{Numerical example}

Consider a composite membrane (as is presented in Fig. 1) with a middle annular segment of small radial size: $\frac{r_{1}-r_{0}}{r_{2}}=0.01$. We assume that the density $\rho_{1}$ of the middle segment is higher than the remaining portion of the membrane: 
$\sigma_{1}=\frac{\rho_{1}}{\rho_{2}}=2 ; 5 ; 10 ; 50 ; 100$ and $\rho_{0}=\rho_{2}$. The frequency parameter $\Omega_{0 i}=\omega_{0 i} r_{2} \sqrt{\frac{\rho_{2}}{s}}$ corresponding to axisymmetric modes of vibration of the composite membrane by using equation (17) are numerically determined. The first four frequency values

$\Omega_{0 i}$ of the membrane versus the location of the middle ring $\frac{r_{0}}{r_{2}}$ are presented in

Figure 3 . The location of the middle ring significantly effects the frequency values of the membrane. The comparison of the results for the results for the various values of $\sigma_{1}$ has shown that the increase of the density of the middle ring causes the decrease of the membrane frequencies except for the discrete values of $\frac{r_{0}}{r_{2}}$.

\section{Conclusions}

The exact solution to the problem of free vibration of a composite circular membrane by using the Green's function method has been presented. The membrane consists of one circular and two annular segments, each of constant density. The derived frequency equation can be used to investigate the step-wise radial changes of the membrane density on the eigenfrequencies of the system. The presented example shows the effect of inside located concentric ring with bigger density as in the remaining portion of the membrane, on the eigenfrequencies of the system. It was found that an increase of density of the distinguished membrane ring causes a decrease of the eigenfrequencies of the membrane system. The further research in this range should concern the application of the presented method in frequency analysis of the membrane consisting of an arbitrary number of membrane rings.

\section{References}

[1] Laura P.A.A., Rossit C.A., La Malfa S., Transverse vibrations of composite, circular annular membranes: exact solution, Journal of Sound and Vibration 1998, 216(1), 190-193.

[2] Rossit C.A., La Malfa S., Laura P.A.A., Antisymmetric modes of vibrations of composite, doubly-connected membranes, Journal of Sound and Vibration 1998, 217(1), 191-195.

[3] Marciniec A., Noga S., Natural frequencies and mode shapes of a composite annular membrane, Vibrations in Physical Systems 2010, 24, 277-282.

[4] Duffy D.G., Green's Functions with Applications, Chapman \& Hall/CRC, Boca Raton, Washington 2001.

[5] Kukla S., Siedlecka U., Zamorska I., Green's functions for interior and exterior Helmholtz problems, Scientific Research of the Institute of Mathematics and Computer Science Czestochowa University of Technology 2012, 1(11), 53-62.

[6] Wolfram S., The Mathematica Book, Wolfram Media, 5th ed., 2003. 